\title{
C-Jun N-terminal kinase (JNK) isoforms play differing roles in otitis media
}

\author{
William Yao ${ }^{1,2}$, Meredith Frie ${ }^{1,2}$, Jeffrey Pan ${ }^{1,2}$, Kwang Pak ${ }^{2,3}$, Nicholas Webster ${ }^{4}$, Stephen I Wasserman ${ }^{5}$ \\ and Allen F Ryan ${ }^{2,3,6^{*}}$
}

\begin{abstract}
Background: Innate immunity and tissue proliferation play important roles in otitis media (OM), the most common disease of childhood. CJUN terminal kinase (JNK) is potentially involved in both processes.

Results: Genes involved in both innate immune and growth factor activation of JNK are upregulated during OM, while expression of both positive and negative JNK regulatory genes is altered. When compared to wildtypes (WTs), C57BL/6 mice deficient in JNK1 exhibit enhanced mucosal thickening, with delayed recovery, enhanced neutrophil recruitment early in $\mathrm{OM}$, and delayed bacterial clearance. In contrast, JNK2 ${ }^{-/-}$mice exhibit delayed mucosal hyperplasia that eventually exceeds that of WTs and is slow to recover, delayed recruitment of neutrophils, and failure of bacterial clearance.

Conclusions: The results suggest that JNK1 and JNK2 play primarily opposing roles in mucosal hyperplasia and neutrophil recruitment early in OM. However, both isoforms are required for the normal resolution of middle ear infection.
\end{abstract}

Keywords: Middle ear, Nontypeable haemophilus influenzae, Infection, MAP kinase signaling, Tissue proliferation, Inflammation

\section{Background}

Otitis media $(\mathrm{OM})$ is one of the most prevalent and costly acute medical problems that afflict children and infants aging from six months to six years [1-3]. Although acute OM may not cause immediate concern in developed countries, persistent and chronic OM produces a sustained conductive hearing loss and may result in irreversible damage to the middle and/or inner ear. In the developing world, in contrast, OM causes an estimated 30,000 deaths per year, and contributes to half of the world's burden of serious hearing loss [4]. Understanding the pathogenesis of $\mathrm{OM}$ is thus an important research goal.

Consequences of OM to the middle ear (ME) include hyperplasia of the ME mucosa with the potential for mucus secretion, leukocytic infiltration into the $\mathrm{ME}$

\footnotetext{
* Correspondence: afryan@ucsd.edu

${ }^{2}$ Departments of Surgery/Otolaryngology, University of California, San Diego, La Jolla, CA 92093, USA

${ }^{3}$ Research Service, Veterans Administration Medical Center, San Diego, CA 92151, USA

Full list of author information is available at the end of the article
}

cavity, and ME effusion; ME inflammation associated with $\mathrm{OM}$ can also cause tissue damage and intense pain. While $\mathrm{OM}$ is multifactorial, one of its most prominent causes is bacterial infection. In particular, as the usage of pneumococcal vaccines has increased, the incidence of OM caused by nontypeable Haemophilus influenzae (NTHi) has risen [5]. The mechanisms by which bacteria influence the ME during $\mathrm{OM}$ is a subject of increasing interest. With respect to the context of the present study, there is extensive evidence that innate immune receptors play a role in the resolution of acute OM e.g. [6], but the downstream effectors of pathogen-induced responses in the ME are not well understood. Both the Toll-like receptor (TLR) and NODlike receptor families of innate immune receptors can signal via CJUN N-terminal kinase (JNK) to activate proinflammatory target gene expression including cytokines and chemokines. However, JNK is also the product of a protooncogene, and like other mitogen activated protein kinases (MAPKs) [7] can be a potent mediator of tissue growth [8]. Finally, JNK is a stress protein and has been shown to mediate apoptosis in some circumstances [9]. 
Elucidating JNK signaling pathways activated during OM would not only improve our understanding of ME immune signaling and its regulation, but might also provide new gene targets for alternative OM treatments. JNK signaling is complex, with numerous upstream and downstream molecules potentially participating. TLR signaling via JNK involves the TLR adaptor MyD88, which activates IRAK4 and TRAF6, in turn activating TAK1. TAK1 phosphorylates the MAP kinase kinases (MAP2Ks) 4 and 7, which in turn activate JNK [10]. Activated JNK phosphorylates members of the JUN family of proteins, including CJUN, JUNB, JUND, as well as ATF2. Phosphorylated CJUN homodimerizes or forms heterdimers with phosphorylated FOS or ATF2, to form the AP-1 transcriptional activator, which can potentially regulate many genes $[11,12]$. Growth factor binding to their cognate receptors recruit adaptors, including SHC, GRB and SOS, can activate the small GTPases KRAS, and CDC42 [13], which in turn activate MAPK kinase kinase kinases (MAP3Ks) 1 and 4. These converge with TLR signaling by activating MAP2Ks 4 and 7 to, in turn, phosphorylate JNK.

Activation of the JNK pathway is also regulated by multiple scaffolding proteins, which function to collect signaling effectors into molecular complexes to regulate signal transduction and localize the complexes to specific parts of the cell. These include the JNK-interacting proteins (JIPs) 1-3, which can aggregate MLK, MAPKs 2 and 7, and JNK to enhance JNK activation. Similarly, POSH (plenty of SH3) interacts with MLK3 to aid in JNK phosphorylation [14] while arrestin $\beta 2$ (ARRB2) has been shown to dock directly to MAP2K4 [15] and activate JNK [16]. The dual specificity phosphatase (DUSP) proteins are scaffolds that influence MAPKs with varying actions and specificity [17]. DUSP1, 2, 8 and 10 positively regulate JNK. DUSP 16 [18], 18, 19 (SKRP1) and 22 inhibit JNK activity $[19,20]$. CDC42EP2 can also inhibit JNK by blocking the activity of upstream CDC42 [21], while the JNK target CJUN can be inhibited by competition with JUNB.

JNK signaling is complicated by the fact that JNK is not one protein. Three JNK genes (Jnk1, Jnk2, Jnk3) can be alternatively spliced into 10 isoforms, which allows for different JNK activities in specific tissue types [22]. The Jnk1 and Jnk2 genes are expressed in tissues throughout the body, but Jnk3 has expression limited primarily to the brain, heart and testes [7]. JNK1 and JNK2 have been implicated as important in immune signaling and evidence has shown that JNK1 and JNK2 protein activation is increased in rat ME mucosa during OM [23].

The JNK1 and JNK2 isoforms have been shown to perform different signaling roles to induce specific physiological changes [22]. However, differences between JNK1 and JNK2 actions are not well characterized, and most JNK effectors are stimulated by both JNK1 and JNK2, making it difficult to determine signaling differences between the two molecules.

The objective of this study was to investigate JNK signaling networks during OM, by documenting the expression of various genes in the JNK signaling cascade during the course of a ME infection, and by comparing OM in mice deficient in JNK1 or JNK2 with that in wildtype (WT) mice. We hypothesized that genes encoding many elements of JNK signaling pathways would be regulated during OM, and that JNK1 and JNK2 would contribute to different aspects of the OM phenotype.

\section{Methods}

\section{Animals}

Experimental methods and protocols were performed on healthy, naive $60-90$ day old ( 25 gm) mice, according to the National Institutes of Health guidelines on the care and use of laboratory animals and approved by the Institutional Animal Care and Use Committee of the San Diego VA Medical Center. Mice were used since acute $\mathrm{OM}$ in this species is quite similar to the disease in humans [3,24], and since gene knockouts are available. Animals were housed under SPF conditions with 4-6 mice per cage, free access to food and water, and environmental enrichment. Gene array studies were performed on C57/CB F1 hybrid mice (Jackson Labs, Bar Harbor, ME), to reduce the influence of the recessive mutations common in inbred mouse strains. Knockout experiments were performed on mice systemically deficient in JNK1 or JNK2 on a C57BL/6 background, and upon C57BL/6 WT controls (bred in-house from Jackson Labs stock), since they were produced on this background. Gene deficient animals are most frequently created in this species/strain. Generating C57/CB F1 hybrids with the KOs would not have been possible. It should be noted that we have performed arrays on C57BL/ 6 mice at 6 and 48 hours after NTHi inoculation, and the results are quite similar to those seen with $\mathrm{C} 57 /$ CB F1 hybrid mice.

\section{Bacterial strain and culture conditions}

Minimally passaged Haemophilus influenzae strain 3655 (nontypeable, biotype II; extracted from the ME of a child with $\mathrm{OM}$ ) was grown, inoculated, and used at a concentration of $10^{5}-10^{6}$ bacteria/ml to generate an inflammatory response within the ME. Inocula were prepared as described by Melhus and Ryan [3].

\section{Surgical procedure}

Surgeries were performed aseptically in a designated area of the laboratory. WT, JNK1 ${ }^{-1-}$, and $\mathrm{JNK} 2^{-/-}$mice were anesthetized with an intraperitoneal injection of 
0.1-0.2 $\mathrm{ml}$ per $30 \mathrm{~g}$ bodyweight of rodent cocktail (13.3 $\mathrm{mg} / \mathrm{ml}$ ketamine HCL, $1.3 \mathrm{mg} / \mathrm{ml}$ xylazine and $0.25 \mathrm{mg} / \mathrm{ml}$ acepromazine), which provides brief, deep anesthesia. MEs were exposed bilaterally by a ventral approach through a midline neck incision. ME bullae were then punctured using a 25-gauge needle. Approximately 5 microliters of NTHi (500-5000 cfu at $10^{5}-10^{6}$ bacteria/ml) were injected into the ME cavity using a 30-gauge needle, which allowed air to exit the ME opening during injection. Excess fluid was soaked up with a sterile cotton swab. The wound was closed and the skin incision was stapled. The mice were injected with the analgesic buprenorphine and lactated Ringer's solution subcutaneously. Mice were examined for fluid leakage into the external ear canal to ensure no penetration of the tympanic membrane had occurred from the 25-gauge needle. Surgeries were timed to permit sacrifice at the appropriate interval. All animals were monitored after surgery until ambulatory, and then daily for health status until sacrifice. No adverse events occurred.

\section{DNA microarray}

Expression of genes involved in JNK signaling was evaluated during the course of an acute episode of OM in WT mice by DNA microarray. For each time point evaluated, twenty WT mice were inoculated with NTHi as above. The ME mucosae were harvested at each of the following intervals: 0, 3 and 6 hours, as well as 1, 2, 3, 5 and 7 days after NTHi inoculation. 0 hour (control) mice received no treatment. The tissue was homogenized in TRIzol $^{\text {TM }}$ (Invitrogen, Carlsbad, CA) and total RNA was extracted. RNA was labeled and hybridized to two Affymetrix MU430 2.0 microarrays. This procedure was then duplicated with an additional 20 mice for each time point, to obtain an independent replication. Gene transcript expression levels were evaluated using variancemodeled posterior inference (VAMPIRE) [25]. Functional gene families were assessed by gene ontogeny (GO) analysis, and specific genes were assessed at individual time points, after Bonferonni correction for multiple tests, using Genespring GX 7.3 (Agilent). Individual gene behavior was evaluated as fold change from expression observed in uninoculated ( 0 hour) control mice.

As a control, mock injection of only saline was performed in identical groups of mice. This also resulted in the significant regulation of some genes. However, they almost invariably exhibited lower levels of regulation than that seen after NTHi inoculation. For the 47 genes reported here, 30 were not regulated by saline injection, and all of the remaining genes were regulated at lower levels, many substantially so (data not shown).

\section{Histology}

OM was evaluated in 3 experimental groups: $33 \mathrm{JNK} 1^{-/-}$ mice, $33 \mathrm{JNK} 2^{-1-}$ mice, and $33 \mathrm{WT}$ control mice with the same genetic background (C57BL/6). JNK knockout and C57BL/6 strains were obtained from Jackson Labs. Each of these groups was randomly subdivided into sets of 3 mice, 1 set for each of 11 time points. When more than one batch of bacteria was used each batch was distributed between all groups. The mice were bilaterally inoculated in the ME with NTHi, and were sacrificed under general anesthesia by intracardiac perfusion with $3 \mathrm{ml}$ of PBS followed by $3 \mathrm{ml}$ of a 4\% paraformaldehyde solution at 0, 6 or 12 hrs or $1,2,3,5,7,10,14$, or 21 days after inoculation. In all experiments, the $0 \mathrm{hr}$ time point represents untreated MEs. The MEs were extracted, postfixed with $4 \%$ PFA overnight, and decalcified in an 8\% EDTA/4\% PFA solution over a 14 day period. The ME bullae were embedded in paraffin and serially sectioned at 7 micrometers. Sections were stained with hematoxylin-eosin and mounted. Images of the ME cavity were digitally recorded and mucosal thickness and percentage area of the $\mathrm{ME}$ lumen that contained inflammatory cells were determined from standardized areas spaced throughout the ME, using 6 MEs per condition, as described previously [26].

To identify the cells present in the ME, highmagnification $(400 \times)$ images were captured for regions with inflammatory cells in the lumen. The number of neutrophils and macrophages present in each 400x image was counted, and counts from 3 images were averaged for each ME. Data from 6 MEs were evaluated per condition.

\section{Bacterial clearance}

The presence of live bacteria in the ME was evaluated in 3 experimental groups: $21 \mathrm{JNK} 1^{-/-}$mice, $21 \mathrm{JNK} 2^{-/-}$ mice, and $21 \mathrm{WT}$ control mice $(\mathrm{C} 57 \mathrm{BL} / 6)$. Each group was randomly subdivided into sets of 3 mice, 1 for each of 7 time points. MEs were extracted from $3 \mathrm{WT}$ and 3 JNK knockouts for each isoform (JNK1 or JNK2) at 1, 2, $3,5,10,14$, and 21 days after ME inoculation. The MEs were opened and a 1-microliter loop was used to obtain a sample from the ME lumen for NTHi culture onto chocolate agar plates with nutrients specific for NTHi growth. For MEs without fluid present, the loop was rubbed across the ME mucosal surface. Each loop was T-streaked onto 4 successive quadrants of each plate. The plates were then analyzed after 24 hours' incubation at $37^{\circ} \mathrm{C}$. The identity of NTHi was verified by plate specificity and by colony color and morphology.

Bacterial data were analyzed by determining the presence or absence of NTHi in each plate, and quantified by counting the number of quadrants in which colonies were observed.

\section{Statistical analysis}

Data were analyzed using Statview (Scientific Computing, Cary, NC). Parametric data were analyzed by ANOVA with 
the Fisher post-hoc test, Bonferroni corrected for multiple comparisons, for tests of individual time points. Leukocyte counts were measured as percentages, and these data were analyzed with the Kruskal-Wallace nonparametric ANOVA equivalent, and the MannWhitney $U$ test for individual time point analysis. All animals in each group were included in the analyses, and no animals were excluded.

\section{Results}

\section{Expression of JNK signaling genes in the ME during OM}

The expression of mRNA transcripts encoding elements of the innate immune and growth factor JNK signaling cascades leading to the activation of JNK were evaluated from ME gene array data generated before and during NTHi-induced OM. As illustrated in Figures 1, 2, 3 and Additional file 1: Table S1, genes encoding many of the elements of JNK signaling were differentially regulated during OM. Figure 1 illustrates the fold changes observed for genes that link innate immune receptors to JNK. In general, gene regulation was moderate and longlasting. Just downstream from the receptor complex, Traf6 and Irak4 exhibited peaks on enhanced expression at 6 and 24 hours after inoculation, while Tak 1 exhibited more sustained up-regulation. Jnk1 and $J n k 2$ were modestly enhanced during ME infection. Jnk3 was not only expressed at much lower initial levels than the other isoforms, but was consistently down-regulated during OM. However, the gene encoding the direct JNK phosphorylation target Cjun was rapidly, vigorously, and persistently up-regulated.
Figure 2 documents the regulation of many of the genes that participate in growth factor activation of JNK. Most of the significantly affected genes were up-regulated, and most peaked at 24 hours after inoculation. Exceptions were $C d c 42$, the most highly up-regulated gene, which peaked at 6 hours, and Map3K1 and Map3K4, which were downregulated in the first hours after NTHi inoculation, but then recovered to pre-inoculation levels.

Figure 3 illustrates the regulation of genes that modulate the JNK signaling pathway. All of the significantly affected positive regulators were up-regulated, most within 3 hours of inoculation. The negative regulators were more variable. Junb was strongly up-regulated at 3 hours and remained elevated until 2 days, while Dusp16 peaked at 1 day. Dusp 18 was strongly down-regulated at 6 hours, while Dusp 19 was slightly up-regulated at 2 days.

While CJUN is the canonical phosphorylation target of the JNKs, many additional targets have been identified, including other potential components of the AP-1 transcriptional complex such as JUNB, JUND, and ATF2, growth regulators and apoptosis-related proteins. The expression of 38 genes encoding JNK phosphorylation targets was assessed during OM (see Additional file 2: Table S2), 18 that are activated by JNK phosphorylation, 18 that are inhibited, and 2 that can be dually affected [27]. Of these, 14 activation genes, 9 inhibition genes and both dual genes were regulated. Up-regulation was most common (20 of 25 genes), but down-regulated genes included the growth suppressor $p 53$ (an activation target) and the apoptosis suppressor Bcl2 (potentially dually affected).

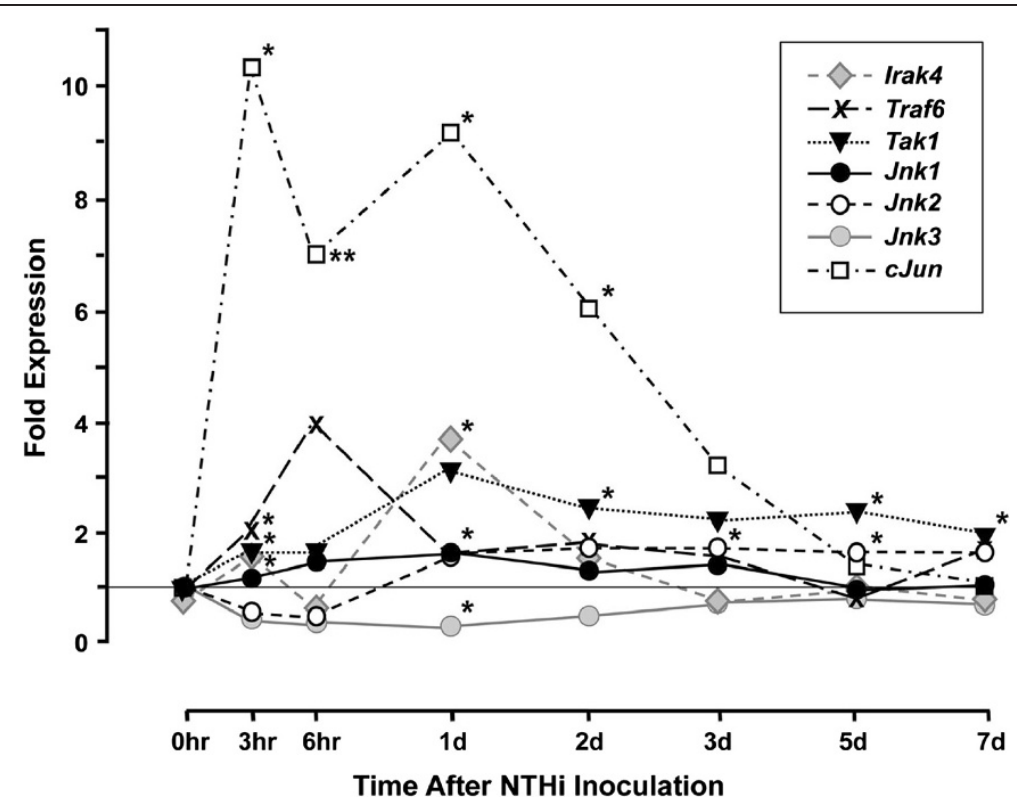

Figure 1 Regulation of JNK signaling genes during OM. ME expression of genes related to the JNK signaling pathway in WT mice during OM, assessed by DNA microarray. Genes involved in TLR JNK signaling. 


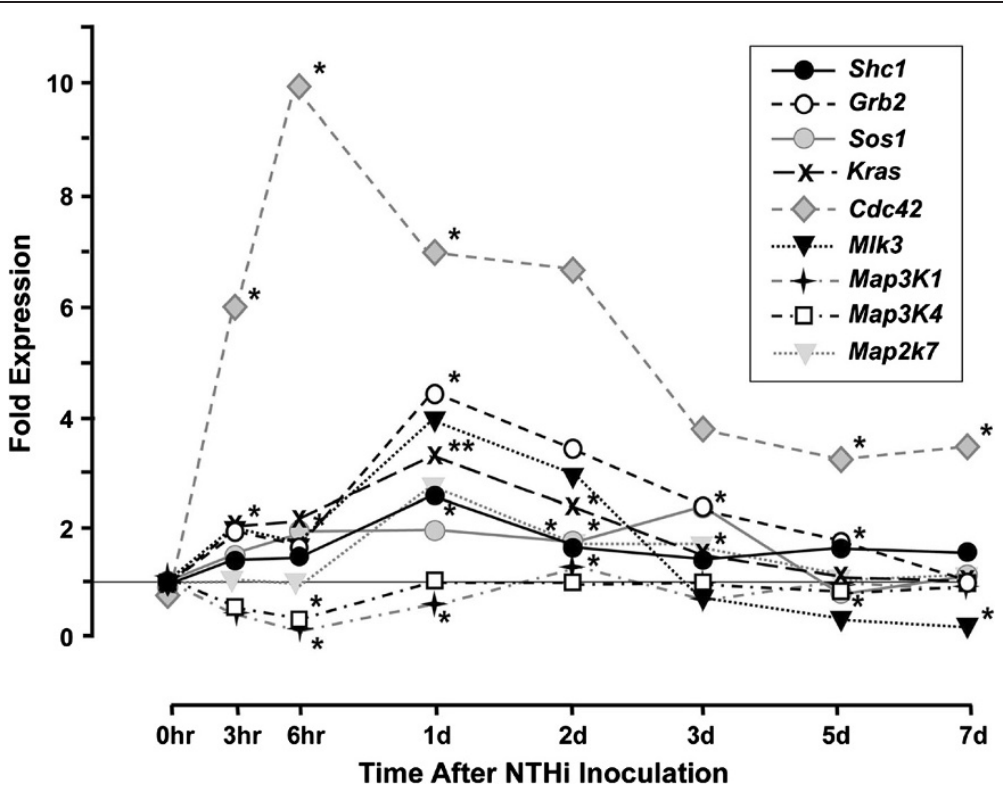

Figure 2 Regulation of JNK signaling genes during OM. ME expression of genes related to the JNK signaling pathway in WT mice during OM, assessed by DNA microarray. Genes invoved in growth factor JNK signaling.

\section{Requirement of JNK1 and JNK2 for normal bacterial} clearance of NTHi infection

WT mice inoculated in the ME with NTHi exhibited positive cultures at $1 \mathrm{~d}, 2 \mathrm{~d}$ and $3 \mathrm{~d}$, with $6 / 6$ MEs being positive in multiple quadrants at $2 \mathrm{~d}$. The degree of bacterial recovery declined regularly from $\mathrm{d} 1$ to $\mathrm{d} 3$. No NTHi colonies were cultured from WT mice on $\mathrm{d} 5$ and thereafter. In contrast, the clearance of bacteria from JNKdeficient mice was greatly delayed. In the case of JNK1 $1^{-1-}$ mice, 4/6 MEs were positive for bacteria on $\mathrm{d} 5$, a low
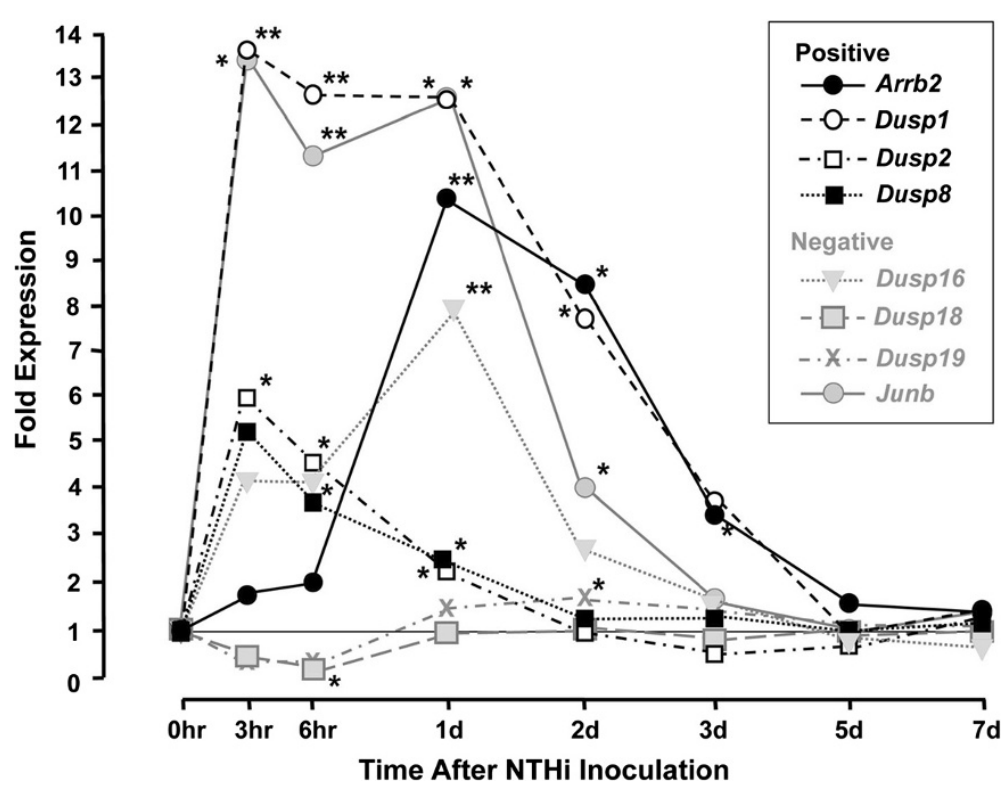

Figure 3 Regulation of JNK signaling genes during OM. ME expression of genes related to the JNK signaling pathway in WT mice during OM, assessed by DNA microarray. Genes involved in JNK signaling regulation. The genes encoding JNK1 and JNK2 were modestly regulated by NTHi infection. Jnk1 was up-regulated for the first few days of OM, while Jnk2 exhibited down-regulation 3-6 hours after inoculation, followed by up-regulation on subsequent days. In contrast, many of the genes up- and down-stream of JNK were strongly regulated. The up-stream JNK activator genes Cdc42, Rac2 and Arrb2, and the down-stream target genes cJun, Stat3 and Stat4, and the negative regulator genes Dusp 16 and Cdc42ep2 were rapidly and persistently up-regulated. ${ }^{*} \mathrm{P}<0.05,{ }^{* *} \mathrm{P}<0.01,{ }^{* * *} \mathrm{P}<0.001$, compared to 0 hour expression. 
degree of bacterial recovery was observed at $10 \mathrm{~d}$ and $14 \mathrm{~d}$, and clearance was not achieved until 21d. In JNK $2^{-1-}$ mice, NTHi were isolated from at least some MEs at all times, including at $21 \mathrm{~d}$, and the degree of bacterial colonization at later times was greater than for $\mathrm{JNK} 1^{-/-}$mice (Table 1 ).

\section{Histology of the ME during OM}

Figure 4 illustrates the mucosal response of WT and JNK-deficient mice to NTHi inoculation of the ME. The ME of WT mice exhibit a characteristic inflammatory response when infected with NTHi [3,24,28,29]. The epithelial and stromal layers of the mucosa increase in thickness, and influxes of neutrophils and macrophages successively enter the ME cavity. In WT mice, these responses peak at d1-3 after inoculation with NTHi, and the $\mathrm{ME}$ returns to its pre-inoculation condition after 7-10 days. In both $\mathrm{JNK} 1^{-/-}$and $\mathrm{JNK} 2^{-/-}$mice, the $\mathrm{ME}$ undergoes mucosal thickening and infiltration of inflammatory cells, with the changes most prominent at $\mathrm{d} 2$ after inoculation. However, in $\mathrm{JNK} 1^{-/-}$mice the $\mathrm{ME}$ failed to return to a pre-inoculation condition until after 10 days. It was also observed that a subset of JNK2 $2^{-/-}$ mice underwent resurgence of inflammation at $\mathrm{d} 7$, but also returned to normal by $\mathrm{d} 10$.

\section{Mucosal thickness}

One measure of inflammation in the ME is the thickness of the mucosa, which displays hyperplasia in response to various inflammatory stimuli [24,29]. Quantitative data on the thickness of the ME mucosal epithelium and stroma are presented in Figures 5, 6, 7. In WT mice, modest growth of the mucosal epithelium (Figure 6) and more substantial expansion of the stroma (Figure 7) was apparent by $1 \mathrm{~d}$ after NTHi infection. Both epithelial and stromal growth peaked at $2 \mathrm{~d}$ and recovered baseline levels by $3-5 \mathrm{~d}$. In JNK $1^{-1-}$ mice, the mucosal epithelium exhibited significant overproliferation at $1 \mathrm{~d}, 2 \mathrm{~d}, 3 \mathrm{~d}, 5 \mathrm{~d}$ and $7 \mathrm{~d}(\mathrm{p} \leq .05)$. The stroma exhibited greater growth at $3 \mathrm{~d}, 5 \mathrm{~d}$, and $7 \mathrm{~d}(\mathrm{p} \leq .05)$. JNK $2^{-/-}$mice initially exhibited delayed growth of both the epithelium and stroma compared to WT mice, with significantly lower thickness at $1 d(p<.05)$. Subsequently, the mucosal epithelium was thicker than in WT mice at $3 \mathrm{~d}, 5 \mathrm{~d}$ and $7 \mathrm{~d}(\mathrm{p} \leq .05)$. The stromal compartment showed significant over proliferation at $2 \mathrm{~d}, 3 \mathrm{~d}$ and $7 \mathrm{~d}(\mathrm{p} \leq .05)$. Interestingly, the epithelium and stroma of $\mathrm{JNK} 2^{-/-}$mice became slightly but significantly less thick than the WT at 10d ( $\leq \leq .05)$.

\section{Leukocyte infiltration of the ME}

In WT mice, the percentage of ME cavity occupied by inflammatory cells peaked at $2 \mathrm{~d}$ and had recovered to baseline levels by $7 d$ (Figure 7 ). When infiltrating cells were further quantified as to cell type, WT mice showed neutrophil infiltration to the ME cavity beginning at $6 \mathrm{~h}$, peaking at $1 \mathrm{~d}$, and returning to baseline levels by $5 \mathrm{~d}$ (Figure 8 ). Macrophage infiltration in WTs peaked at $2 \mathrm{~d}$ and returned to baseline levels by $7 d$ (Figure 9 ).

In $\mathrm{JNK}^{-/-}$mice, the percentage of the ME occupied by inflammatory cells was similar to that observed in WTs, with the exception that a somewhat lower proportion of cells was seen at $5 \mathrm{~d}(\mathrm{p} \leq .01)$ (Figure 7$)$. When cell infiltration was evaluated for cell type, JNK $1^{-/-}$neutrophil infiltration slightly but significantly exceeded that seen in WTs at $6 \mathrm{~h}(\mathrm{p} \leq .05)$, was dramatically higher at $1 \mathrm{~d}$ and modestly higher at $7 \mathrm{~d}(\mathrm{p} \leq .01)$ (Figure 8$)$.

JNK $2^{-1-}$ mice showed substantially greater inflammatory cell infiltration into the ME cavity at $2 \mathrm{~d}$ and $7 \mathrm{~d}$ $(\mathrm{p} \leq .05)$, and a slight but significant decrease at $6 \mathrm{~h}$ (Figure 7). JNK2 ${ }^{-/-}$neutrophil infiltration was significantly increased at $7 \mathrm{~d}(\mathrm{p} \leq .01)$ (Figure 8$)$, while macrophage infiltration was dramatically increased at $2 \mathrm{~d}(\mathrm{p} \leq .01)$ (Figure 9).

\section{Discussion}

Regulation of JNK signaling genes supports a role in the host response to ME infection

As seen in the microarray data for the ME mucosa, many of the genes that are involved in the JNK pathway

Table 1 Bacterial clearance

\begin{tabular}{lllllll}
\hline $\begin{array}{l}\text { Time after } \\
\text { NTHi inoculation }\end{array}$ & $\begin{array}{l}\text { WT \# } \\
\text { of culture } \\
\text { positive plates }\end{array}$ & $\begin{array}{l}\text { WT } \\
\text { Quadrants } \\
\text { positive in culture } \\
\text { positive plates }\end{array}$ & $\begin{array}{l}\text { JNK1 } \\
\text { \# of culture } \\
\text { positive plates }\end{array}$ & $\begin{array}{l}\text { JNK1 } \\
\text { Quadrants positive } \\
\text { in culture positive } \\
\text { plates }\end{array}$ & $\begin{array}{l}\text { JNK2 }^{-1-} \\
\text { \# of culture } \\
\text { positive plates }\end{array}$ & $\begin{array}{l}\text { JNK2 }^{-/-} \\
\text {Quadrants positive } \\
\text { in culture positive } \\
\text { plates }\end{array}$ \\
\hline Day 1 & $4 / 6$ & 4.00 & $5 / 6$ & 3.20 & $3 / 6$ & 2.33 \\
Day 2 & $6 / 6$ & 3.00 & $2 / 6$ & 3.00 & $4 / 6$ & 2.75 \\
Day 3 & $3 / 6$ & 1.00 & $4 / 6$ & 2.50 & $5 / 6$ & 2.80 \\
Day 5 & $0 / 6$ & 0.00 & $4 / 6$ & 2.00 & $2 / 6$ & 1.50 \\
Day 10 & $0 / 6$ & 0.00 & $3 / 6$ & 1.00 & $1 / 6$ & 4.00 \\
Day 14 & $0 / 6$ & 0.00 & $2 / 6$ & 1.00 & $1 / 6$ & 1.00 \\
Day 21 & $0 / 6$ & 0.00 & $0 / 6$ & 0.00 & $2 / 6$ & 2.50 \\
\hline
\end{tabular}

No NTHi colonies were isolated from the ME for WT mice, from 5 days after infection onward. In JNK1 ${ }^{-/-}$mice, NTHi were isolated up to day 14 . In JNK2 ${ }^{-/-}$mice, NTHi was isolated throughout the 21 day observation period. 


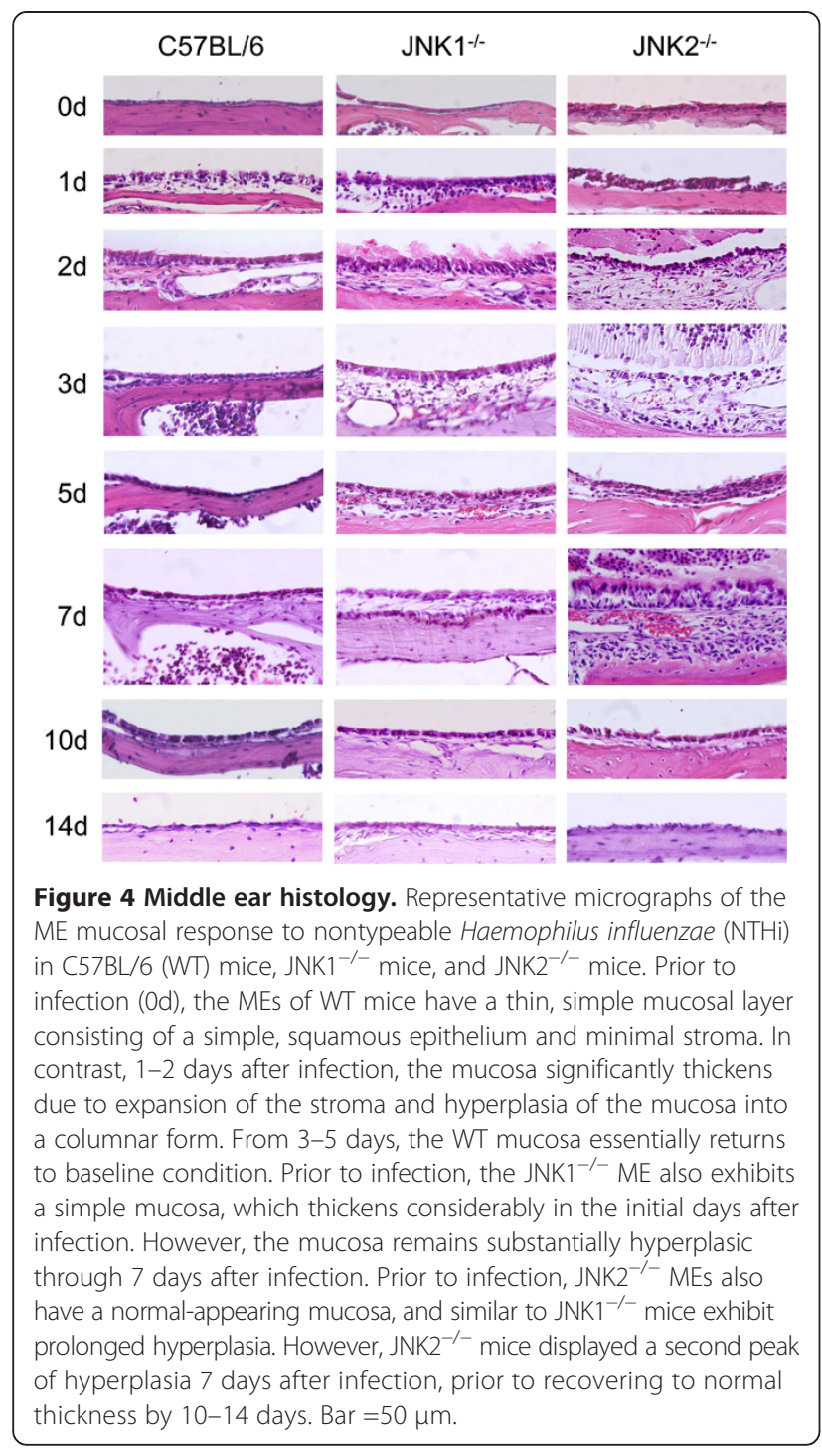

show significant regulation during the course of OM. This degree of regulation suggests that the pathway plays a critical role in this condition. It is significant that the up-regulation of most genes peaked at 1d after inoculation. ME inflammation, leukocytic infiltration and mucosal hyperplasia all peaked at $2 \mathrm{~d}$ after inoculation. Given the delays to be expected in protein synthesis and cellular responses, the increase in gene expression related to JNK signaling is well positioned to participate in all three manifestations of OM.

It should be noted that we observed only modest regulation of the genes encoding JNK1 and JNK2 themselves. This suggests that these signaling molecules are maintained at functional levels in the resting ME, and are predominantly regulated post-transcriptionally, perhaps by phosphorylative activation. This is consistent with prior data at the protein level demonstrating little change in total JNK during OM, but increased phospho-JNK1 and

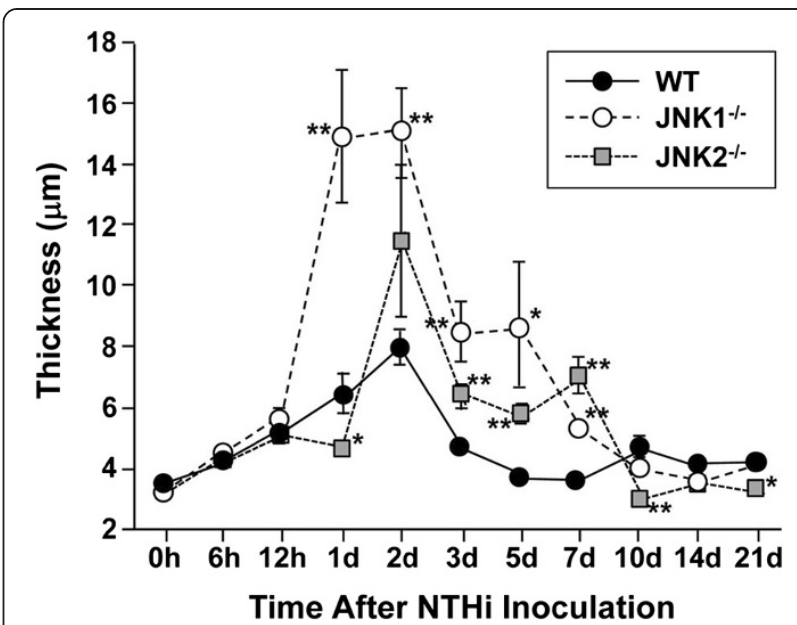

Figure $\mathbf{5}$ ME mucosal hyperplasia. Evaluation of thickness of the ME mucosal epithelium of WT (filled circles), JNK1 ${ }^{-1-}$ (open circles) and $\mathrm{NKK}^{-/-}$(gray squares) mice after NTHi inoculation.

-JNK2 [23]. In contrast, the genes encoding signaling molecules upstream from JNK (e.g. IRAK4, CDC42, KRAS) were up-regulated during $\mathrm{OM}$, suggesting increased involvement of JNK signaling during the course of OM. Again, protein data confirms a peak of phospho-JNK at $72 \mathrm{~h}$ after initiation of infection [23]. We also found that genes encoding both positive (e.g. ARRB2, RAC2) and negative (e.g. CDC42EP2, DUSP16) regulators of the JNK pathway showed significant up-regulation during OM, suggesting tight regulation of JNK activity, perhaps to prevent bystander injury to host tissues from inflammation and/or apoptosis [30].

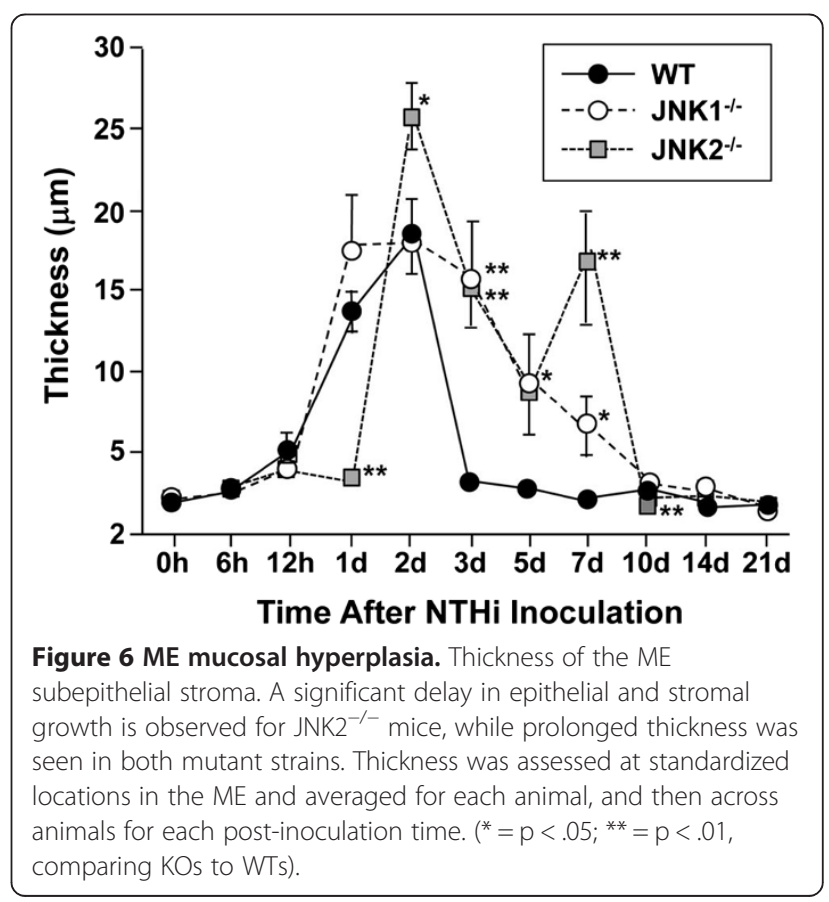




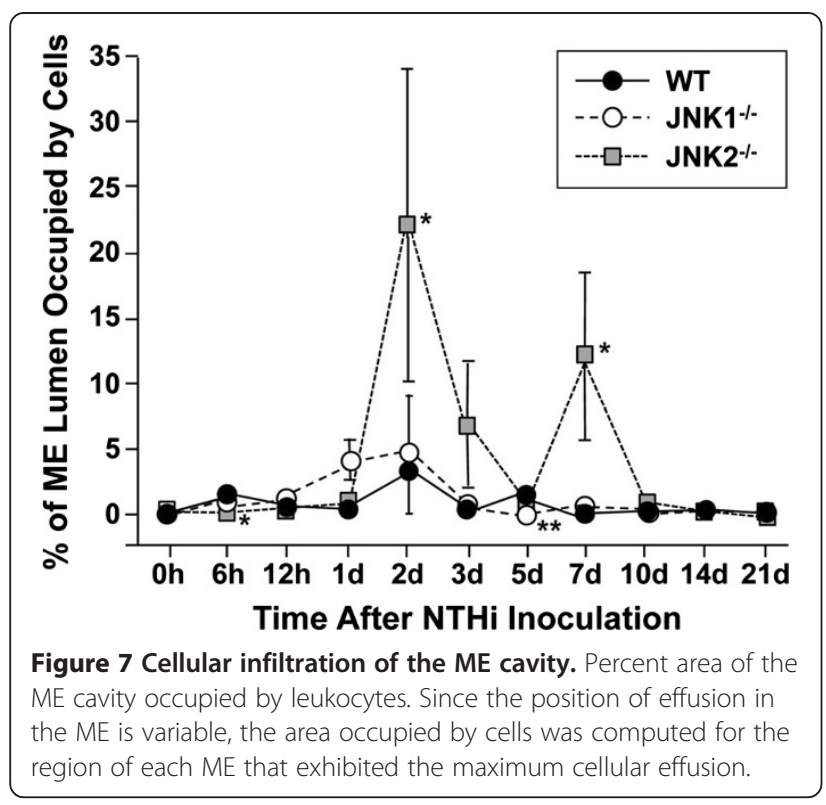

The gene encoding the primary downstream target of JNK, CJUN, showed very strong and early upregulation. CJUN dimerizes with members of the JUN, FOS or ATF2 subfamilies to create AP-1 [31], a multifunctional transcription factor. AP-1 induces the production of multiple pro-inflammatory cytokines, including tumor necrosis factor alpha (TNFA) [32]. TNFA is a proinflammatory mediator important in innate immunity. It is strongly expressed during $\mathrm{OM}$ and $\mathrm{TNFA}^{-1-}$ mice have shown delayed NTHi-induced OM resolution [28]. This suggests that the JNK pathway is critical in inducing inflammation, which in turn is critical for proper recruitment and access of immune cells to the site of infection for infection clearance. This is

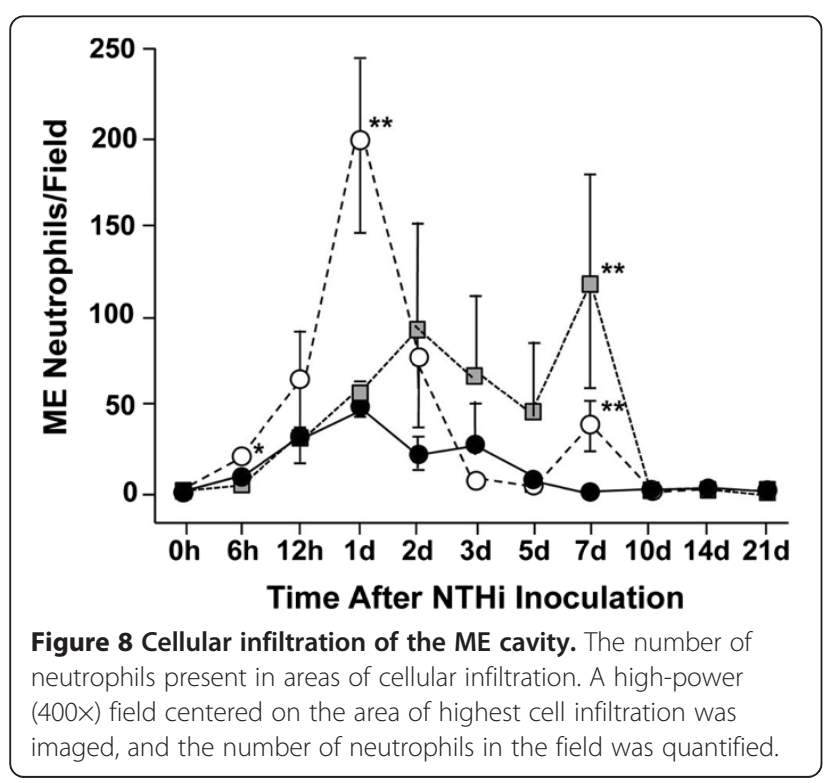

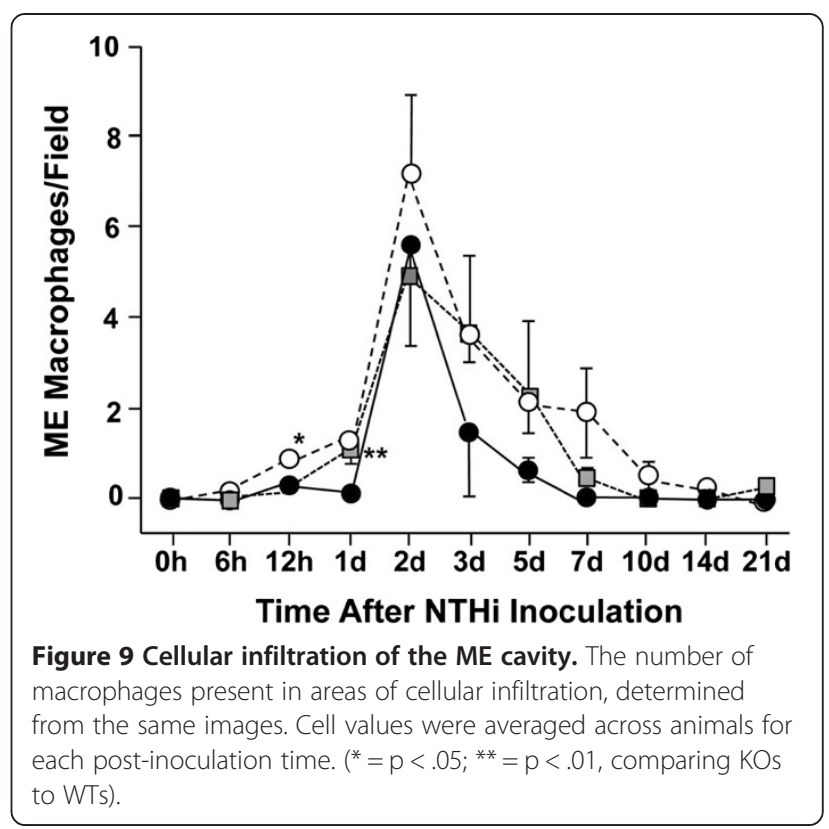

consistent with the delayed bacterial clearance observed in both JNK1- and JNK2-deficient mice. AP-1 is also implicated in tissue proliferation [33]. Its primary components, CJUN and CFOS, are both positive regulators of tissue growth and their active heterodimers can cause anchorage-independent proliferation [34]. The up-regulation of GF-related JNK signaling genes is consistent with a role for JNK in tissue proliferation during OM. The regulation of a majority of genes encoding direct JNK phosphorylation targets involved in AP-1 transcription, tissue proliferation and apoptosis $[11,35,36]$ further implicates the molecules of the JNK signaling system in OM.

JNK deletion indicates that both isoforms play a role in OM Both JNK1 ${ }^{-/-}$and $\mathrm{JNK}^{-/-}$mice showed significantly different physiological responses to NTHi infection of the ME when compared to WT animals. Both mutants exhibited delayed bacterial clearance, as well as atypical leukocyte recruitment. Both $\mathrm{JNK} 1^{-1-}$ and $\mathrm{JNK} 2^{-/-}$also showed a delayed return to baseline mucosal thickness. Interestingly, both $\mathrm{JNK}^{-/-}$and $\mathrm{JNK} 2^{-/-}$MEs showed evidence of a resurgence of $\mathrm{OM}$ at $7 \mathrm{~d}$, after the initiation of recovery. The similarity of these changes suggests that JNK1 and JNK2 are both necessary for a normal innate immune response to $\mathrm{OM}$, and that some functions of the two isoforms appear to overlap.

Albeit delayed, eventual resolution of infection was observed in all $\mathrm{JNK}^{-/-}$mice and in most $\mathrm{JNK}^{-/-}$mice. Moreover, the basic histopathologic features of OM, although altered, were present in both strains, and both mutants showed return of mucosal thickness and 
leukocyte levels to baseline. This suggests redundancy between the two isoforms and/or JNK-independent mechanisms that operate in parallel with JNKdependent processes to mediate pathogenesis and recovery from OM. NFkB, known to mediate innate immune responses in addition to JNK, is a strong candidate for such a parallel pathway [37].

We did not obtain evidence that an individual JNK isoform is responsible for hyperplasia of the ME mucosa, since neither JNK1- nor JNK2-deficient animals exhibited a consistently decreased proliferative response to bacterial infection. Rather, the proliferative response was more persistent, presumably reflecting prolonged bacterial infection. Since we have previously shown that a pan-JNK inhibitor reduces mucosal proliferation during OM [23], our current results suggest redundancy between the two isoforms. Of course, JNK-independent pathways may also be involved. Growth factor stimulation can activate alternative growth pathways mediated by the extracellular signal-regulated kinases (ERK) and p38 MAP kinases, both of which have been shown to be activated during OM [38,39], and as above NFKB is another potential contributory pathway.

\section{JNK1 and JNK2 have distinct roles in regulating the ME response to NTHi}

Although the mechanistic differences remain unclear, it is known that JNK1 and JNK2 are not necessarily interchangeable in the JNK signaling pathway. This is also apparent in our data, since the two isoforms were unable to completely compensate for each other during $\mathrm{OM}$ and since the ME phenotypes of the two mutants were distinct. JNK1-deficient mice exhibited earlier growth of the mucosal epithelium compared to the WT. In contrast, JNK2 ${ }^{-1-}$ mice exhibited a delay in the growth of both the mucosal epithelium and stroma. JNK1 ${ }^{-1-}$ mice exhibited greatly enhanced neutrophil recruitment early in OM, while JNK2deficient mice exhibited a delay in neutrophil recruitment and greater persistence of neutrophils. JNK2 ${ }^{-1-}$ mice also showed enhanced macrophage recruitment to the ME, while $\mathrm{JNK}^{-1-}$ mice did not. The phenotype of JNK2 $2^{-/-}$ mice suggests a more serious defect in inflammation than seen in JNK1 $1^{-/-}$mice, given the delays in mucosal proliferation and leukocyte recruitment and the greater persistence of bacterial infection in these mice. This may reflect the reported higher levels of phosphorylated JNK2 detected in the ME after bacterial inoculation, when compared to JNK1 [23].

The fact that JNK1-deficient mice exhibit enhanced neutrophil recruitment and epithelial thickness early in OM could reflect a role for this isoform in limiting inflammation during early OM. Supporting this possibility, JNK1 has been found to be protective against ischemic injury in the brain [40].

\section{Implications and limitations of the study}

Drawing conclusions from animal studies regarding human disease must, of course, be performed with caution. Although the characteristics of NTHi-induced OM in mice are quite similar to those observed in humans (e.g. $[3,29])$, differences in genetics and in the response of mice to a human pathogen must be considered in the interpretation of data. With this caveat, our findings have implications for the treatment of OM. The use of JNK inhibitors alone to treat OM would probably not be advisable, given the possibility of reduced bacterial clearance. However, our data suggest that, in combination with antibiotics, JNK inhibition has the potential to reduce inflammation and mucosal hyperplasia. Any such strategy would need to take into account differences the roles of JNK1 and JNK2 in OM. Thus, a JNK2-specific inhibitor might be more beneficial than a pan-JNK inhibitor.

\section{Conclusions}

In summary, we found that JNK signaling plays a significant role in $\mathrm{OM}$, as reflected by altered expression of JNK signaling genes, as well as reduced bacterial clearance and altered ME phenotype in mice deficient in JNK isoforms. JNK1 and JNK2 are both required for the normal resolution of NTHi infection in the ME. JNK1 and JNK2 appear to play both redundant and distinct roles in OM, with JNK2 mediating the early recruitment of neutrophils and being more critical for bacterial clearance. JNK1 may actually limit inflammation early in $\mathrm{OM}$. The results have implications for JNK inhibition as a therapeutic option in OM.

\section{Additional files}

Additional file 1: Table S1. Change in expression of JNK signaling genes during acute $\mathrm{OM}$.

Additional file 2: Table S2. Change in expression of JNK target genes during acute $\mathrm{OM}$.

\section{Abbreviations}

CSOM: Chronic suppurative OM; DUSP: Dual specificity phosphatase; ERK: Extracellular signal-regulated kinases; GO: Gene ontogeny; JIPs: JNKinteracting proteins; JNK: C-Jun N-terminal Kinase; MAP2Ks: MAP kinase kinases; MAP3K: MAPK kinase kinase kinases; MAPKs: Mitogen activated protein kinases; ME: Middle ear; NTHi: Nontypeable Haemophilus influenzae; OM: Otitis media; POSH: Plenty of SH3; TLR: Toll-like receptor; TNFA: Tumor necrosis factor alpha; VAMPIRE: Variance-modeled posterior inference; WT: Wildtype.

\section{Competing interests}

The authors declare that they have no competing interests.

\section{Authors' contributions}

WY and MF contributed equally to the completion of this work. WY, MF, JP, KP and AFR performed the animal experiments, histological evaluations and data analysis. NW and AFR performed the gene array analysis. WY, MF, JP, SIW and AFR wrote the manuscript. AFR designed and supervised the study. All authors read and approved the final manuscript. 


\section{Acknowledgments}

This work was entirely supported by the National Institutes of Health/ National Institute of Deafness and Other Communication Disorders (NIH) NIDCD) grants DC000129, DC006279 and by the Research Service of the VA.

\section{Author details}

${ }^{1}$ Departments of Biology, University of California, San Diego, La Jolla, CA 92093, USA. ²Departments of Surgery/Otolaryngology, University of California, San Diego, La Jolla, CA 92093, USA. ${ }^{3}$ Research Service, Veterans Administration Medical Center, San Diego, CA 92151, USA. ${ }^{4}$ Departments of Medicine Endocrinology and Metabolism, University of California, San Diego, La Jolla, CA 92093, USA. 5 Departments of Medicine/Allergy \& Immunology, University of California, San Diego, La Jolla, CA 92093, USA. ${ }^{\text {SSurgery/ }}$ Neuroscience, 0626, UCSD School of Medicine, 9500 Gilman Drive, La Jolla, CA 92093-0626, USA

Received: 12 February 2014 Accepted: 2 October 2014 Published online: 14 October 2014

\section{References}

1. Infante-Rivard C, Fernandez A: Otitis media in children: frequency, risk factors, and research avenues. Epidemiol Rev 1993, 15:444-465.

2. Klein JO: The burden of otitis media. Vaccine 2000, 1:S2-S8.

3. Melhus A, Ryan AF: A mouse model for acute otitis media. APMIS 2003, 111:989-111994

4. World Health Organization: Chronic suppurative otitis media: burden of illness and management options. http://www.who.int/pbd/deafness/ activities/hearing_care/otitis_media.pdf.

5. Leibovitz E, Jacobs MR, Dagan R: Haemophilus influenzae: a significant pathogen in acute otitis media. Pediatr Infect Dis J 2004, 23:1142-1152.

6. Leichtle A, Lai Y, Wollenberg B, Wasserman SI, Ryan AF: Innate signaling in otitis media: pathogenesis and recovery. Curr Allergy Asthma Rep 2011, 11:78-84.

7. Davis RJ: Signal transduction by the JNK group of MAP kinases. Cell 2000, 103(2):239-252.

8. Potapova O, Gorospe M, Bost F, Dean NM, Gaarde WA, Holborrk NJ: c-Jun $\mathrm{N}$-terminal kinase is essential for growth of human T98G glioblastoma cells. J Biol Chem 2000, 275:24767-24775.

9. Lin A: Activation of the JNK signaling pathway: breaking the brake on apoptosis. Bioessays 2003, 25:17-24

10. Haeusgen W, Herdegen T, Waetzig $V$ : The bottleneck of JNK signaling: molecular and functional characteristics of MKK4 and MKK7. Eur J Cell Biol 2011, 90:536-544.

11. Karin M, Liu ZG, Zandi E: AP-1 function and regulation. Curr Opin Cell Biol 1997, 9:240-246.

12. Foletta VC, Segal DH, Cohen DR: Transcriptional regulation in the immune system: all roads lead to AP-1. J Leukoc Biol 1998, 63:139-152.

13. Fanger $\mathrm{GR}$, Johnson $\mathrm{NL}$, Johnson GL: MEK kinases are regulated by EGF and selectively interact with Rac/Cdc42. EMBO J 1997, 16:4961-4972.

14. Xu Z, Kukekov NV, Greene LA: POSH acts as a scaffold for a multiprotein complex that mediates JNK activation in apoptosis. EMBO J 2003, 22:252-261

15. Li X, Macleod R, Dunlop AJ, Edwards HV, Advant N, Gibson LC, Devine NM, Brown KM, Adams DR, Houslay MD, Baillie GS: A scanning peptide array approach uncovers association sites within the JNK/beta arrestin signalling complex. FEBS Lett 2009, 583:3310-3316.

16. Lattin J, Zidar DA, Schroder K, Kellie S, Hume DA, Sweet MJ: G-proteincoupled receptor expression, function, and signaling in macrophages. J Leukoc Biol 2007, 82:16-32.

17. Jeffrey KL, Camps M, Rommel C, Mackay CR: Targeting dual-specificity phosphatases: manipulating MAP kinase signaling and immune responses. Nat Rev Drug Discov 2007, 6:391-403.

18. Masuda K, Shima H, Watanabe M, Kikuchi K: MKP-7, a novel mitogenactivated protein kinase phosphatase, functions as a shuttle protein. J Biol Chem 2001, 276:39002-39011.

19. Ho DT, Bardwell AJ, Grewal S, Iverson C, Bardwell L: Interacting JNK-docking sites in MKK7 promote binding and activation of JNK mitogen-activated protein kinases. J Biol Chem 2006, 281:13169-13179.

20. Zama T, Aoki R, Kamimoto T, Inoue K, Ikeda Y, Hagiwara M: A novel dual specificity phosphatase SKRP1 interacts with the MAPK kinase MKK7 and inactivates the JNK MAPK pathway. Implication for the precise regulation of the particular MAPK pathway. J Biol Chem 2002, 277:23909-23918.

21. Hirsch DS, Pirone DM, Burbelo PD: A new family of Cdc42 effector proteins, CEPs, function in fibroblast and epithelial cell shape changes. J Biol Chem 2001, 276:875-883.

22. Gupta S, Barrett T, Whitmarsh AJ, Cavanagh J, Sluss HK, Derijard B, Davis RJ: Selective interaction of JNK protein kinase isoforms with transcription factors. EMBO J 1996, 15:2760-2770.

23. Furukawa M, Ebmeyer J, Ryan AF, Ebmeyer U, Sudhoff H, Broide D, Ryan AF, Wasserman S: Jun N-terminal protein kinase enhances middle ear mucosal proliferation during bacterial otitis media. Infect Immun 2007, 75:2562-2571

24. Ryan AF, Ebmeyer J, Furukawa M, Pak K, Melhus A, Waserman SI, Chung WH: Mouse models of induced otitis media. Brain Res 2006, 1091:3-8.

25. Hsiao A, Ideker T, Olefsky JM, Subramaniam S: VAMPIRE microarray suite: a web based platform for the interpretation of gene expression data. Nucleic Acids Res 2005, 33:W627-W632.

26. Ebmeyer J, Furukawa M, Pak K, Ebmeyer U, Sudhoff H, Broide D, Ryan AF, Wasserman S: Role of mast cells in otitis media. J Allergy Clin Immunol 2005, 116:1129-1135.

27. Bogoyevitch MA, Kobe B: Uses for JNK: the many and varied substrates of the c-Jun N-terminal kinases. Microbiol Mol Biol Rev 2006, 70:1061-1095.

28. Leichtle A, Hernandez M, Ebmeyer J, Yamasaki K, Lai Y, Radek K, Choung YH, Euteneuer S, Pak K, Gallo R, Wasserman SI, Ryan AF: CC chemokine ligand 3 overcomes the bacteriocidal and phagocytic defect of macrophages and hastens recovery from experimental otitis media in TNF-/- mice. $\mathrm{J}$ Immunol 2010, 184:3087-3097.

29. Hernandez M, Leichtle A, Pak K, Ebmeyer J, Euteneuer S, Obonyo M, Guiney DG, Webster NJ, Broide DH, Ryan AF, Wasserman SI: Myeloid differentiation primary response gene 88 is required for the resolution of otitis media. $\mathrm{J}$ Infect Dis 2008, 198:1862-1869.

30. Triantafilou M, Triantafilou K, Lipopolysaccharide recognition: CD14, TLRs and the LPS-activation cluster. Trends Immunol 2002, 23:301-304.

31. Chiu R, Boyle WJ, Meek J, Smeal T, Huner T, Karin M: The c-Fos protein interacts with c-Jun/AP-1 to stimulate transcription of AP-1 responsive genes. Cell 1988, 54:541-552

32. Levy CS, Slomiansky V, Gattelli A, Nahmod K, Pelisch F, Blaustein M, Srebrow A, Coso OA, Kordon EC: Tumor necrosis factor alpha induces LIF expression through ERK1/2 activation in mammary epithelial cells. J Cell Biochem 2010, 110:857-865.

33. Shaulian E, Karin M: AP-1 as a regulator of cell life and death. Nat Cell Biol 2002, 4:E131-E136.

34. van Dam H, Huguier S, Kooistra K, Baguet J, Vial E, van der Eb AJ, Herrlich P, Angel P, Castellazzi M: Autocrine growth and anchorage independence: two complementing Jun-controlled genetic programs of cellular transformation. Genes Dev 1998, 12(8):1227-1239.

35. Bode AM, Dong Z: The functional contrariety of JNK. Mol Carcinog 2007, 46:591-598

36. Leppä S, Bohmann D: Diverse functions of JNK signaling and c-Jun in stress response and apoptosis. Oncogene 1999, 18:6158-6162.

37. Kumar H, Kawai T, Akira S: Pathogen recognition by the innate immune system. Int Rev Immunol 2011, 30:16-34

38. Palacios SD, Pak K, Kayali AG, Rivkin AZ, Aletsee C, Melhus A, Webster NJ, Ryan AF: Participation of Ras and extracellular regulated kinase in the hyperplastic response of middle-ear mucosa during bacterial otitis media. J Infect Dis 2002, 186:1761-1769.

39. Palacios SD, Pak K, Rivkin AZ, Kayali AG, Austen D, Aletsee C, Melhus A, Webster NJ, Ryan AF: Role of p38 mitogen-activated protein kinase in middle ear mucosa hyperplasia during bacterial otitis media. Infect Immun 2004, 72:4662-4667.

40. Brecht S, Kirchhof R, Chromik A, Willesen M, Nicolaus T, Raivich G, Wessig J, Waetzig V, Goetz M, Claussen M, Pearse D, Kuan CY, Vaudano E, Behrens A, Wagner E, Flavell RA, Davis RJ, Herdegen T: Specific pathophysiological functions of JNK isoforms in the brain. Eur J Neurosci 2005, 21:363-377.

doi:10.1186/s12865-014-0046-z

Cite this article as: Yao et al:: C-Jun N-terminal kinase (JNK) isoforms play differing roles in otitis media. BMC Immunology 2014 15:46. 